\title{
ESTUDO DAS MANIFESTACÕES NEUROLÓGICAS EM 93 DOENTES COM INTOXICAÇÃO EXÓGENA POR SUBSTANCIAS QUIMICAS NÃO MEDICAMENTOSAS
}

\author{
S. AGAPEJEV * \\ I. VASSILIEV ** \\ M. M. F. LIMA ***
}

A exposição prolongada a agentes químicos tóxicos utilizados como produtos domésticos, agrícolas e industriais tem sido descrita como causadora de quadros neurológicos $4,5,10,12,18,19,21,23,26,27$, dependendo do tipo de absorção, tempo de exposição, forma de intoxicação 20,21 e da sensibilidade individual 24,28 . A existência da parede protetora do endotélio capilar, formada pelos astrócitos envolvendo a microcirculação, e a baixa concentração proteica existente no líquido cefalorraqueano (LCR) constituem os principais fatores que impedem a entrada de muitas substâncias tóxicas em condiçōes apreciáveis no cérebro. Além disso, a efetividade da barreira hêmato-encefálica (BHE) é variável de uma área para outra, sendo mais permeável ou pelo menos com maior fluxo sanguíneo, no córtex cerebral, no núcleo lateral do hipotálamo, na área postrema, na pineal e no lobo posterior da hipófise 13,22,25. Consequentemente, apenas aqueles tóxicos que não necessitam de ligação proteica e os lipossolúveis dependentes do coeficiente de partição lípide/água têm acesso fácil ao encéfalo. A circulação do $\mathrm{LCR}$, as vilosidades aracnóideas, a BHE e os transportes ativos existentes são as vias de eliminação desses produtos do sistema nervoso central (SNC).

Os metais pesados (MP) geralmente atuam como competidores dos oligoelementos, são hidrossolúveis e agem como substâncias protoplasmáticas produzindo lesão celular. São encontrados, principalmente o arsênico: como contaminantes de alguns inseticidas, predominantemente os organoclorados; como integrantes de tintas, colas, solventes; como poluentes de água, de alguns alimentos (por exemplo o açúcar), do ar e de cigarros 30 . Levam a lesão reversível do sistema nervoso periférico (SNP), com regressão lenta, que pode durar meses ou anos. Os organoclorados (OC) são altamente lipossolúveis, atingindo o SNC com grande frequência, principalmente o cerebelo. São integralmente absorvidos e formam resíduos que permanecem acumulados no tecido gorduroso corpóreo por mais de 30 anos; possuem também ação espoliativa de cálcio e neurotropismo sobre as células de Schwann, principalmente das fibras não mielinizadas 21. A exposição prolongada aos OC pode também resultar em

Universidade Estadual Paulista, Campus de Botucatu: * Departamento de Neurologia e Psiquiatria, Faculdade de Medicina (FMB); ** Departamento de Farmacologia, Instituto Básico de Biologia Médica e Agrícola (IBBMA); *** Académica, FMB. 
efeitos oncogênicos, mutagênicos e teratogênicos, que surgem até muitos anos após ter existido a exposiçāo crônica 16. As substâncias organofosforadas (OF) caracterizam-se pelo alto gráu de toxicidade e pela ação predominantemente parassimpatomimética, bloqueando a acetilcolinesterase e outras esterases - segundo Amitai e col., citados por Alonso e col. ${ }^{3}$ — inclusive as do sistema nervoso 14,15 . Existem evidências que sugerem ser a esterase neurotóxica o primeiro local de ação dos ésteres OF neurotóxicos 8 . Estes ésteres fosforilam as proteinas cerebrais desenvolvendo lesão tissular que se inicia quando mais de $70 \%$ dessas proteinas istejam fosforiladas, diminuindo bastante os locais de ligação. Aldridge \& Barnes 1,2 mostram que não há correlação entre a proteina inibidora dos $O F$ ( esterases neurotóxicas (proteinas cerebrais fosforiladas $=$ proteinas neurotóxicas). Duffy e col.7 consideram que os possíveis efeitos farmacológicos dos OF sobre o SNC sejam: o aumento de acetilcolina que, pela diminuição da colinesterase tissular, levaria a alteraçôes crônicas na morfologia sináptica e organização bioquímica; as elevadas concentrações de acetilcolina durante o tempo de exposição produziriam mudanças nos receptores pós-sinápticos do SNC, tornando-os mais sensiveis à acetilcolina endógena; a exposição a ésteres OF leva a diminuição do fluxo sanguineo cerebral, produzindo hipóxia. Provavelmente, estes são também os mecanismos responsáveis pelos quadros de hipersensibilizaçāo que ocorrem nas intoxicações pelos OF. Os carbamatos (Cb), embora nāo sejam altamente perigosos como agentes neurotóxicos, podem provocar alterações neurológicas e comportamentais sem que haja suspeita clinica de intoxicação. A sua toxicidade deve-se a inibiçăo das colinesterases do sistema nervoso, eritrócitos e plasma que, por sua vez, inibiram outras esterases tissulares. A natureza dessas esterases tissulares permanece ainda obscura. Há, portanto, semelhança entre os mecanismos de ação dos $\mathrm{OF}$ e dos $\mathrm{Cb} 9$. Os herbicidas (Hb) determinam lesão celular com precipitação de proteinas, produzindo frequentes quadros de hipersensibilizaçāo 9,17. Os efeitos agudos são semelhantes aos provocados pelo álcool, atuando como depressores do SNC em doses altas, produzindo lesăo parcial da BHE por comprometimento capilar e levando a acúmulo do produto no SNC 31. São poucos os estudos a respeito de herbicidas e por isso ainda permanecem pouco esclarecidos os seus mecanismos de ação no SNP e no SNC. Assim como o álcool, os $\mathrm{Hb}$ têm comportamento variável, afetando tanto o SNC como o SNP, dependendo da grande diferença de susceptibilidade individual ao envenenamento 24. São encontrados como contaminantes do solo, água e ar. Embora a BHE seja o local menos permeável do organismo ao: tíxicos. estes a atravessam em quantidades razoáveis devido à variabilidade na necessidade de aporte nutritivo entre as diversas áreas cerebrais e à lipossolubilidade que favorece sua livre passagem para o SNC. O processo de eliminação faz-se pela diurese, bile, respiração, fezes e/ou vômitos, saliva, sudorese e circulação do LCR. Consequentemente, as intoxicaçōes crônicas podem produzir quadros clínicos bastante variados, tanto pelo comprometimento sistêmico como pela lesão do sistema nervoso, levando a dificuldade diagnóstica da etiologia tóxica, fundamentalmente quando nāo se valoriza a procedência e a profissão do doente, nem o avanço livre e desenfreado da tecnologia quimica industrial. 
Este trabalho tenta mostrar a importância dos produtos tóxicos, principalmente os inseticidas agrotóxicos, na gênese de patologias do sistema nervoso, enfatizando as do SNC.

\section{CASUÍSTICA E MÉTODOS}

No período de agosto de 1981 a maio de 1984 foram realizados exames toxicológicos em 132 doentes provenientes de Ambulatórios e Enfermarias do Hospital das Clínicas da FMB, sendo selecionados 93 que apresentavam sinais e/ou sintomas sugestivos de comprometimento do sistema nervoso. Destes, em 12 a pesquisa foi feita em amostras de sangue, urina e LCR; em 30 no sangue e LCR; em 51, apenas no sangue. Todos os pacientes apresentavam antecedentes de contato e/ou ingestāo de substância tóxica em alguma etapa de sua vida, procediam de locais potencialmente tóxicos ou apresentavam quadros clínicos sugestivos de intoxicação exógena aguda ou crônica, ou apresentavam patologias sem etiologia definida.

A pesquisa de substâncias tóxicas foi realizada no Departamento de Farmacologia do IBBMA, utilizando-se: método semi-quantitativo de cromatografia em camada delgada de sílica-gel para a pesquisa de inseticidas organoclorados, organofosforados, organoclorofosforados (OCF), carbamatos e herbicidas; cromatografia em papel de filtro, para metais pesados; método analítico quantitativo específico para os fenóis (F). Simultaneamente foram realizadas comparaçōes com padrões de concentraçāo conhecida. Em dois pacientes não se conseguiu precisar os grupos constituintes dos adubos (A) pelas técnicas de extraçāo de inseticidas utilizadas ou a identificaçāo com os padrōes conhecidos.

\section{RESULTADOS}

Dos 132 doentes estudados, aproximadamente $70 \%$ apresentavam diagnóstico sindrômico neurológico e em $30 \%$ não se detectou qualquer sinal ou sintoma de afecça do sistema nervoso (Tabela 1), observando-se nestes um predominio de manifestacires

\begin{tabular}{|c|c|}
\hline ôrgão ou Sistema & Número de Pacientes \\
\hline Sistema nervoso central (SNC) & $59(44,8 \%)$ \\
\hline Sistema nervoso periférico (SNP) & $34(25,7 \%)$ \\
\hline Trato gastro-intestinal & $12(9 \%)$ \\
\hline Sistema hematológico & $5(4 \%)$ \\
\hline Pele & $4(3 \%)$ \\
\hline Trato urinário & $2(1,5 \%)$ \\
\hline Sistema cárdio-respiratório & $2(1,5 \%)$ \\
\hline Mal definidas * & $14(10,5 \%)$ \\
\hline Total & $132(100 \%)$ \\
\hline
\end{tabular}

Tabela 1 - Distribuição dos 132 doentes segundo a forma de manifestação clínica em relugão ao orgāo ou sistema comprometido: (*) inclui: mal-estar, vertigem, nauseas. dores no corpo, ansiedade, irritabilidade. 
gastro-intestinais, hematológicas e dermatológicas. Dos 93 pacientes neurológicos (Tabela 2), cerca de $37 \%$ apresentavam manifestações de afeç̧ão do SNP e $63 \%$ do SNC. Destes, aproximadamente $23 \%$ apresentavam epllepsta; $11 \%$, sindrome de hipertensão intracraniana e quadro clinico de comprometimento central do sistema piramidal manifesto por paresia e/ou hiperreflexia; $6 \%$ tinham sinais de disfunção cortical predominantemente do lobo frontal; $5 \%$ mostravam sindrome cerebelar; $4 \%$, sindrome meningea por sangramento espontaneo e, os $3 \%$ restantes, sindrome pseudobulbar, sindrome vestibular e sindrome do cordajo posterior. Obitos ocorreram em 8 dos 93 doentes $(8,6 \%)$ : por ingestão acidental em um (criunça de 6 anos de idade, ingestăo de raticida doméstico), por tentativa de suicidio em dois (adultos, lavradores, ingestáo de formicida e Aldrin). Os outros 5 pacientes referiam contacto com agrotóxicos e em dois deles desenvolveu-se quadro de hipersensibilização manifestado em um por encefalite necrotizante multifocal e no outro por granulomatose alérgica, ou sindrome de Churg-Strauss; nos demais, as patologias observadas foram: intoxicação por herbicidas, carcinomatose de sistema nervoso e edema cerebral (Tabela 3). Os grupos tóxicos isolados que predominaram, com suas manifestaçōes patológicas (Tabela 5) em ordem decrescente de frequiência, foram: $\mathrm{MP}, 30 \%$ (neurite periférica, epilepsia, radiculoneurite, comprometimento do sistema piramidal, sindrome de hlpertensão intracranlana e sindrome cortical frontal); $\mathrm{OC}, 11,8 \%$ (neurite periférlca, sindrome de hipertensão intracraniana e epllepsia, sindrome cortical, sindrome pseudobulbar, radiculite); OF, $8,6 \%$ (epilepsia e sindromes cortical, cerebelar, meníngea e neurite periférica); Hb, 2,1\% (epilepsia e síndrome de hipertensão intracraniana). Em $17 \%$ não se detectou qualquer das substâncias tóxicas pesquisadas (Tabela 4), apesar da forte referência de contato pregresso com agrotóxicos e, muitas vezes, quadros de intoxicaçåo aguda anteriormente $\dot{a}$ instalação das queixas que motivaram a primeira consulta. o sistema nervoso foi mais comprometido que os demais órgãos (Tabela 6), ocorrendo prevalência de afeçăo, do SNC em relação go SNP, pelos OF e Hb em associação com outras substâncias ou isoladamente.

Forma de Manifestaçăo

Número de Pactentes

Epilepsia.

Hipertensåo intracraniana

Sindrome piramidal

Disfunção cortical

Sindrome cerebelar

Hemorragia meningea espontanea

Sindrome cordonal posterior

Paralisia pseudobulbar

Sindrome vestibular

Neuropatia periférica

Radiculoneurite

Radiculite

$$
\begin{aligned}
21 & (22,6 \%) \\
10 & (10,7 \%) \\
10 & (10,7 \%) \\
6 & (6,4 \%) \\
5 & (5,4 \%) \\
4 & (4,3 \%) \\
1 & (1,1 \%) \\
1 & (1,1 \%) \\
1 & (1,1 \%) \\
25 & (26,8 \%) \\
6 & (6,5 \%) \\
3 & (3,2 \%) \\
93 & (100 \%)
\end{aligned}
$$

Tabela 2 - Distribuiça de 98 pacientes com comprometimento do sistema nervoso segundo a forma de manifestaço no SNC ou SNP. 


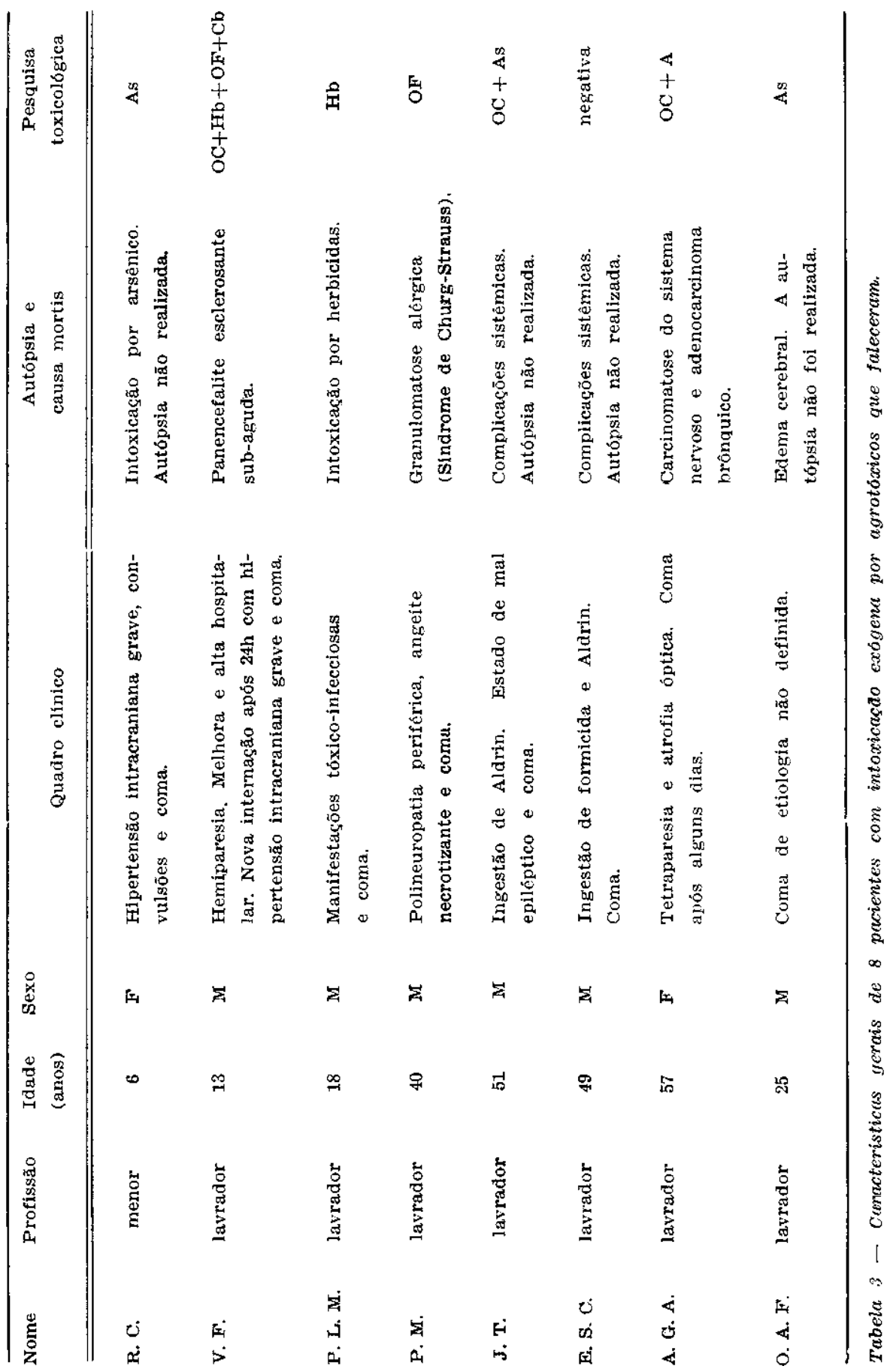




\begin{tabular}{|c|c|c|c|c|}
\hline $\begin{array}{c}\text { Pesquisa } \\
\text { toxicológica }\end{array}$ & $\mathrm{SNC}$ & SNP & Outros* & Total \\
\hline negativa & 11 & $\mathbf{5}$ & 7 & $23 \quad(17 \%)$ \\
\hline positiva & 48 & 29 & 32 & $109(83 \%)$ \\
\hline Total & 59 & 34 & 39 & $132(100 \%)$ \\
\hline
\end{tabular}

Tabela 4 - Distribuicão dos resultados da pesquisa toxicológica, segundo a detecçác (positiva) ou não (negativa) de substancias tóxicas nos manifestacões de $S N C$, SNP $t$ outros orgāos; (*) inclui: sintomas mal definidos, sistema cárdio-respiratorio, trato gastro-intestinal, trato urinario, sistema hematológico e pele.

\begin{tabular}{|c|c|c|c|}
\hline \multirow{3}{*}{$\begin{array}{l}\text { Quadro clínico } \\
= \\
\text { Epilepsia } \\
(n=21)\end{array}$} & \multirow{2}{*}{ 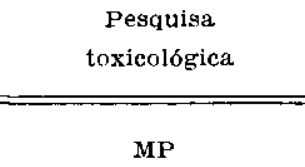 } & \multicolumn{2}{|c|}{$\begin{array}{l}\text { Nümero de } \\
\text { pacientes }\end{array}$} \\
\hline & & 7 & $(7,4 \%)$ \\
\hline & OF & 4 & $(4,2 \%)$ \\
\hline & $\mathrm{OO}$ & 1 & $(1,1 \%)$ \\
\hline & $\mathrm{Hb}$ & 1 & $(1,1 \%)$ \\
\hline & $\mathrm{OC}+\mathrm{MP}$ & 2 & $(2,1 \%)$ \\
\hline & $\mathrm{OC}+\mathrm{OF}$ & 1 & $(1.1 \%)$ \\
\hline & $\mathrm{OC}+\mathrm{Hb}$ & 1 & $(1,1 \%)$ \\
\hline & $O C+O F-t^{-A}$ & 1 & $(1,1 \%)$ \\
\hline & $\mathrm{OC} \cdot \mathrm{OF}+\mathrm{Hb}$ & 1 & $(1,1 \%)$ \\
\hline & negativa & 2 & $(2,1 \%)$ \\
\hline Hipertensão & MP & 2 & $(2,1 \%)$ \\
\hline intracraniana & $\mathrm{OC}$ & 2 & $(2,1 \%)$ \\
\hline \multirow[t]{5}{*}{$(n=10)$} & $\mathrm{Hb}$ & 1 & $(1,1 \%)$ \\
\hline & $\mathrm{OC}+\mathrm{OF}$ & 1 & $(1,1 \%)$ \\
\hline & $\mathrm{OC}+\mathrm{OCF}$ & 1 & $(1,1 \%)$ \\
\hline & $\mathrm{OC}+\mathrm{OF}+\mathrm{Cb}+\mathrm{Hb}$ & 1 & $(1,1 \%)$ \\
\hline & negativa & 2 & $(2,1 \%)$ \\
\hline Sindrome & MP & 3 & $(3,2 \%)$ \\
\hline piramidal & $\mathrm{OC}+\mathrm{MP}$ & 1 & $(1,1 \%)$ \\
\hline \multirow[t]{4}{*}{$(n=10)$} & $\mathrm{OC}+\mathrm{OF}$ & 1 & $(1,1 \%)$ \\
\hline & $\mathrm{OC}+\mathrm{A}$ & 1 & $(1,1 \%)$ \\
\hline & $\mathrm{OC}+\mathrm{OF}+\mathrm{MP}$ & 1 & $(1,1 \%)$ \\
\hline & negativa & 3 & $(3,2 \%)$ \\
\hline
\end{tabular}

Tabela 5 - Distribuiça dos 93 pacientes com quadro clinico nentologico segundo o tipo de manifestaça $e$ a pesquisa toxicotogica encontrada; $n$, número de pacientes. 


\begin{tabular}{|c|c|c|c|}
\hline \multirow{3}{*}{$\begin{array}{l}\text { Quadro clínico } \\
\text { Disfunção cortical } \\
\text { principalmente }\end{array}$} & \multirow{2}{*}{$\frac{\begin{array}{c}\text { Pesquisa } \\
\text { toxicológica }\end{array}}{\text { MP }}$} & \multicolumn{2}{|c|}{$\begin{array}{c}\text { Número de } \\
\text { pacierites }\end{array}$} \\
\hline & & 1 & $(1,1 \%)$ \\
\hline & OC & 1 & $(1,1 \%)$ \\
\hline lobofrontal & OF & 1 & $(1,1 \%)$ \\
\hline \multirow[t]{3}{*}{$(n=6)$} & $\mathrm{OC}+\mathrm{MP}$ & 1 & $(1,1 \%)$ \\
\hline & $\mathrm{OC}+\mathrm{OF}+\mathrm{Cb}$ & 1 & $(1,1 \%)$ \\
\hline & negativa & 1 & $(1,1 \%)$ \\
\hline Sindrome & OF & 1 & $(1,1 \%)$ \\
\hline cerebelar & $\mathrm{OC}+\mathrm{MP}$ & 1 & $(1,1 \%)$ \\
\hline \multirow[t]{2}{*}{$(n=5)$} & $\mathrm{OC}+\mathrm{OCF}$ & 1 & $(1,1 \%)$ \\
\hline & negativa & 2 & $(2,1 \%)$ \\
\hline Hemorragia meníngea & $\mathrm{OF}$ & 1 & $(1,1 \%)$ \\
\hline espontanea & $\mathrm{OC}+\mathrm{MP}$ & 1 & $(1,1 \%)$ \\
\hline \multirow[t]{3}{*}{$(n=4)$} & $\mathrm{OC}+\mathrm{OF}$ & 1 & $(1,1 \%)$ \\
\hline & $\mathrm{Cb}+\mathrm{MP}$ & 1 & $(1,1 \%)$ \\
\hline & negativa & 0 & \\
\hline \multicolumn{4}{|l|}{ Sindrome cordonal posterior } \\
\hline Paralisia pseudobulbar $(n=1)$ & $\infty$ & 1 & $(1,1 \%)$ \\
\hline Sindrome vestibular $(n=1)$ & $\mathrm{OC}+\mathrm{MP}$ & 1 & $(1,1 \%)$ \\
\hline Neuropatia & $\mathrm{MP}$ & 11 & $(11,8 \%)$ \\
\hline periférica & $\mathrm{OC}$ & 5 & $(5,3 \%)$ \\
\hline \multirow[t]{5}{*}{$(n=25)$} & $\mathrm{OF}$ & 1 & $(1,1 \%)$ \\
\hline & $O C+O F$ & 2 & $(2,1 \%)$ \\
\hline & $\mathrm{OC}+\mathrm{Cb}$ & 1 & $(1,1 \%)$ \\
\hline & $\mathrm{OCF}+\mathrm{MP}$ & 1 & $(1,1 \%)$ \\
\hline & negativa & 4 & $(1,3 \%)$ \\
\hline Radiculoneurite & $\mathbf{M P}$ & 4 & $(4,3 \%)$ \\
\hline \multirow[t]{2}{*}{$(n=6)$} & $O C+M P$ & 2 & $(2,1 \%)$ \\
\hline & negativa & 0 & \\
\hline Radiculite & $\mathrm{OC}$ & 1 & $(1,1 \%)$ \\
\hline \multirow[t]{2}{*}{$(n=3)$} & $\mathrm{OC}+\mathrm{MP}$ & 1 & $(1,1 \%)$ \\
\hline & negativa & 1 & $(1,1 \%)$ \\
\hline TOTA L & & 93 & $(100 \%)$ \\
\hline
\end{tabular}




\begin{tabular}{|c|c|c|c|c|c|}
\hline Substâncias tóxicas & $\mathrm{SNC}$ & SNP & Outras & & Total \\
\hline MP & 13 & 15 & 20 & 48 & $(44 \%)$ \\
\hline $\mathrm{OC}$ & 5 & 6 & 3 & 14 & $(12,8 \%)$ \\
\hline OF & 7 & 1 & 2 & 10 & $(9,2 \%)$ \\
\hline $\mathrm{Hb}$ & 2 & 0 & 0 & 2 & $(1,8 \%)$ \\
\hline $\mathrm{Fl}$ & 0 & 0 & 1 & 1 & $(0,9 \%)$ \\
\hline $\mathrm{OC}+\mathrm{MP}$ & 7 & 3 & 1 & 11 & $(10,1 \%)$ \\
\hline $\mathrm{OC}+\mathrm{OF}$ & 4 & 2 & 3 & 9 & $(8,2 \%)$ \\
\hline $\mathrm{OC}+\mathrm{OCF}$ & 2 & 0 & 0 & 2 & $(1,8 \%)$ \\
\hline $\mathrm{OC}+\mathrm{Hb}$ & 1 & 0 & 1 & 2 & $(1,8 \%)$ \\
\hline $\mathrm{OC}+\mathrm{Cb}$ & 0 & 1 & 0 & 1 & $(0,9 \%)$ \\
\hline $\mathrm{OC}+\mathrm{A}$ & 1 & 0 & 0 & 1 & $(0,9 \%)$ \\
\hline $\mathrm{MP}+\mathrm{Cb}$ & 1 & 0 & 0 & 1 & $(0,9 \%)$ \\
\hline$M P+O C F$ & 0 & 1 & 0 & 1 & $(0,9 \%)$ \\
\hline $\mathrm{OC}+\mathrm{OF}+\mathrm{MP}$ & 1 & 0 & 0 & 1 & $(0,9 \%)$ \\
\hline $\mathrm{GC}+\mathrm{OF}+\mathrm{A}$ & 1 & 0 & 0 & 1 & $(0,9 \%)$ \\
\hline $\mathrm{OC}+\mathrm{OF}+\mathrm{Cb}$ & 1 & 0 & 0 & 1 & $(0,9 \%)$ \\
\hline $\mathrm{OC}+\mathrm{OF}+\mathrm{Hb}$ & 1 & 0 & 0 & 1 & $(0,9 \%)$ \\
\hline $\mathrm{OC}+\mathrm{OF}+\mathrm{Cb}+\mathrm{Hb}$ & 1 & 0 & 0 & 1 & $(0,8 \%)$ \\
\hline $\mathrm{OC}+\mathrm{OF}+\mathrm{Cb}+\mathrm{OCF}$ & 0 & 0 & 1 & 1 & $(0,9 \%)$ \\
\hline TOTAL & 48 & 29 & 32 & 109 & $(100 \%)$ \\
\hline
\end{tabular}

Tiabela 6 - Distribuigảo dos 109 pacientes com pesquisa toxicológica positiva segundo a substancia detectuda e a manifestaça a apresentuda: MP, metal pesado (um ou mais, com predominio de arsenico e chumbol: $O C$, organoclorado; OF, organofosforado; $O C F$, organoclorofosforado; $\mathrm{Hb}$, herbicida; $\mathrm{Cb}$, carbamato; $\mathrm{Fl}$, fenol; $A$, adubo.

\section{COMENTARIOS}

O sistema nervoso é preferencialmente atingido que outros órgãos. Além disso, o SNC é mais frequentemente lesado que o SNP, apesar da proteção da BHE. O grande teor lipidico do tecido nervoso, a alta lipossolubilidade das substâncias tóxicas, muitas delas não necessitando de ligação com proteinas para se difundir, e as noções 8 de que a primeira açăo dos OF seria relacionada às esterases neurotóxicas, associadas a sensibilidade individual maior, justificam um dano e tropismo maior ao SNC. Embora a literatura 4,11,21,27,29,32 tenha dado ênfase ao quadro neurológico periférico, isolado ou associado ao central, há poucos relatos referentes às sequelas e lesōes no SNC, que costumam estar associadas a alteraçōes psíquicas 6,18 e possuem prognóstico pior que as do SNP 19, coincidindo com nossas observaçöes.

Verificamos que o SNC sofre mais a ação dos $\mathrm{OF}$, isoladamente ou em combinação com os $\mathrm{OC}$ e/ou MP, que o SNP. Este é mais frequentemente danificado pelos MP e OC. Os $\mathrm{Hb}$ mostraram nitida preferência a nivel de $\mathrm{SNC}$, que nos parece ser a consequência de sua ação semelhante ao álcool, embora o número de doentes de nossa casuística seja pequeno $e$, provavelmente, com 
susceptibilidade maior a este grupo químico. A ação dos MP sobre o SNP comprova os dados amplamente descritos na literatura. Dos três casos conı manifestação de lesão do SNP por intoxicação crônica pelos OF, um apresentava síndrome de Churg-Strauss e os outros dois permaneceram com graves sequelas deficitárias nos membros atingidos.

Essa preferência das substâncias tóxicas pelo sistema nervoso é consequência, também, da grande sensibilidade neuronal a qualquer estimulo nocivo produzindo, lesões definitivas e irreversíveis, sem que haja qualquer coisa que possa minimizar ou impedir o processo desde que se instale. Essa lesão neuronal seria a consequência da ação proteolitica sobre a membrana celular, levando a indução da falência do neurônio. Esta, após certo tempo, leva a dano estrutural irreversivel 14, passando por fase de déficit nutricional celular e transformando a sobrevivência das células que exigiriam maior demanda metabólica 5 . Nem mesmo a administração de tiamina durante o periodo latente previne o inicio das manifestaçóes neurológicas (Fenton, citado por Aldridge e Barnes), embora possua ação terapêutica importante como coadjuvante na regeneração das fibras nervosas nas neurites periféricas tóxicas. Os corticóides não atuam diretamente sobre os mecanismos de toxicidade, mas sobre os efeitos inflamatórios e hipersensibilizantes dos produtos, além de agirem como estímulo para ingestão maior de alimentos - conforme Glees e Janzik, referidos por fohnson 15 - melhorando, consequentemente, o aporte nutritivo proteico e energético. Consideramos os nossos dados alarmantes e tentamos mostrar a necessidade de maiores conhecimentos e pesquisas no campo das intoxicações crônicas por substâncias quimicas não medicamentosas, principalmente quanto à elucidação dos mecanismos de ação de tais produtos no SNC e quanto a melhores perspectivas terapêtticas.

Em conclusão, é possivel considerar que: 1) as manifestaçōes neurológicas das intoxicações crônicas são mais frequentes que as alteraçōes sistêmicas, caracterizando-se por grande variabilidade do quadro clínico; 2) há predomínio das manifestações por maior comprometimento do SNC; 3) a epilepsia, como sinal de lesão do SNC, é uma das manifestações mais comuns, e a neurite periférica, como a do SNP; 4) os herbicidas e os organofosforados parecent apresentar tropismo maior para o SNC; 5) a hipersensibilização parece ser um dos mecanismos de ação desses agentes químicos no $\mathrm{SNC}$; 6) os organoclorados e metais pesados afetam tanto o SNC como o SNP, sem apresentar tropismo; 7) as intoxicações crônicas sāo tão graves, ou até mais que as agudas, pois produzem severas sequelas em pessoas na fase mais produtiva da vida; 8 ) as substâncias químicas não medicamentosas são agentes etiológicos que não devem ser relegados em neurologia.

\section{RESUMO}

Foram analisadas as manifestaçōes neurológicas em 93 doentes com suspeita de intoxicação exógena. A deteç̧ão das substancias tóxicas foi realizada no sangue, urina e liquido cefalorraqueano. Verificou-se nitido predomínio do comprometimento do sistema nervoso central em relação ao periférico e aparente tropismo de herbicidas e organofosforados para o sistema nervoso central, com- 
parando-se o comportamento dos metais pesados e organoclorados. São analisados os prováveis mecanismos de ação dessas substancias no sistema nervoso e são apresentados os sinais e/ou sintomas que foram observados.

\section{SUMMARY}

Neurologic manifestations in 93 patients with exogenous intoxication by non-medicamentous chemical substances.

Ninety-three patients with neurological manifestations and suspected exogenous intoxication were analysed. The detection of toxic substances was performed in the blood, urine and cerebrospinal fluid. There was clear predominance of the central nervous system impairment than the peripheric, and an apparent tropism of herbicides and organophosphates to the central nervous system compared with the peripheral nervous systen, and considering the manifestations caused by carbamates and organochlorines. The authors analyse some probable action mechanism of these substances in the nervous system, and show the most frequently neurological signs and symptoms found.

\section{REFERENCIAS}

1. ALDRIDGE, W.N. \& BARNES, J.M. - Neurotoxic and biochemical properties of gome triaryl phosphates. Biochem. Pharmacol. 6:177, 1961.

2. ALDRIDGE, W.N. \& BARNES, J.M. - Further observations on the neurotoxicity of organophosphorus compounds. Biochem. Pharmacol. 15:541, 1966.

3. ALONSO, J.L.; ATALla, A.; CAVALIERE, M.J.; GAGIOTI, S.M. \& LORENTI, M.A.A. - Polineuropatia por Parathion: estudo clínico, eletrofisiológico e histológico de um caso. Arq. Neuro-Psiquiat. (São Paulo) 41:292, 1983.

4. ALLEN, N.; MENDELL, J.R.; BILlMAIER, D.J.; FONTAIN, R.E. \& O'NEILL, J. - Toxic polyneuropathy due to methyl n-buthyl ketone: an industrial outbreak. Arch. Neurol. $32: 209,1975$.

5. CAVANAGH, J.B. - Toxic substances and the nervous system. Brit. med. Bull, $25: 268,1969$.

6. DILLE, J.R. \& SMITH, P.W. - Central nervous system effects of chronic exposure to organophosphate inseticides. Aerosp. Med. 35:475, 1964.

7. DUFFY, F.H.; BUKCHFIELD, J.I.; BARTELS, P.H.; GAON, M. \& SLM, V.M. Long-term effects of an organophosphate upon the human electroencephalogram. Toxicol. appl. Pharmacol. $47: 161,1979$.

8 ECOBICHON, D.J - OrEanophusphorus ester inseticides. In D.J. Ecobichor \& R.M. Joy: Pesticides and Neurological Discases. CRC Press Florida, 1982.

9. ECOBICHON, D.J. - Carbamic acid ester pesticide. In D.J. Ecobichon \& R.M. Joy: Pesticides and Neurological Dišnases. Press Florida. $198 \mathbf{2}$.

10. FORNAZZARI, L. WILKINSON, D.A. ; KAPUR, B.M. \& CARLEN, P.L. - Cerebelar. cortical and functfonal impairment in toluene abusers, Acta neurol. scand. 67:319, 1983.

11. GARLAND, T.O. \& PATTERSON, M.W.H. - Six cases of acrylamide poisoning. Brit. med. J. $4: 134,1967$.

12. HAYMAKER, W.; GINZLER, A.M. \& FERGUSSON, R.L. - The toxic effects of prolonged ingestion of DDT on dogs with special reference to lesions in the brain. Amer. J. med. Sci. 212:423, 1946.

13. HAWKINS, R.A. \& BIEBUYCK, J.F. - Ketone bodies are selectively used by individual brain regions. Science 205:325. 1979.

14. JOHNSON, M.K. - Delayed neurotoxic action of some organophosphorus compounds. Brit. med. Bull. 25:231, 1969. 
15. JOHNSON, M.K. - Irreversible phosphorylation of brain neurotoxic esterase. Monogr. Neurol, Sci. 9:99. Karger, Basel, 1980.

16. JOY, R.M. - Chlorinated hydrocarbon inseticide. In D.J. Ecobichon \& R.M. Joy (eds.): Pesticides and Neurological Diseases. CRC Press Florida, 1982.

17. JUNG, H.D. \& WOLF, F. - Contact eczemas due to forestry use of the herbicide SELEST 100. Dtsch. Gesundheit. 32:1464, 1977.

18. JUNTUNEN, J.; ANTTI-POIKA, M.; TOLA, S. \& PARTANEN, T. - Clinical prognosis of patients with diagnosed chronic solvent intoxication. Acta Neurol. scand. $65: 488,1982$.

19. JUNTUNEN, J.; HERNEBERO, S.; EISTOLA, P. \& HUPIL, V. - Exposure to industrial solvents and brain atrophy. Eur. Neurol. 19:366, 1980.

20. KLAASSEN, C.D. - Absorption, distribution and excretion of toxicants. In J. Doul, C.D. Klaassen \& M.O. Amdur (eds.): Toxirology: The Basic Science of Poisons. Ed. 2. MacMillan, New York, 1981.

21. MARTINEZ, A.J.; TAYLOR, J.R.: DYCK, P.J.; HOUFF, S.A. \& ISAACS, E. Chlordecone intoxication in man. Neurology 28:631, 1978.

22. MATA, M.; FINK, D.J. \& GAINER, H. - Metabolic mapping of functional activity in the hypothalaphysical system of the rat. Science 205:723, 1979.

23. REPKO, J.D. \& CORUM, C.R. - Avaliaçāo e retrospectiva crítica das sequelas neurológicas e comportamentais em razão da absorção de chumbo inorgânico. Rev. bras. Saúde ocupac. 12:7, 1984.

24. ROSENBERG, J. - 2,4-dichlorophenoxyacetic acid (2,4-D): evaluation of human health hazards. Hazard Alert System, Epidemiological Studies Laboratory, Berkeley, 1980.

25. SHEA, P.A. \& APRISON, M.H. - The distribution of acetyl - CoA in specific areas of the CNS of the rat as measured by a modification of a radio-enzymatic assay for acetylcholine and choline. J. Neurochem. 28:51, 1977.

26. SPENCER, P.S.; SCHAUMBURG, H.H.; RALEIGH, R.L. \& TERHAAR, C.J. Nervous system degeneration produced by the industrial solvent methyl $n$-buty] ketone. Arch. Neurol. 32:219, 1975.

27. TAYLOR, J.R.; SELHORST, J.B.; HOUFF, S.A. \& MARTINEZ, A.J. - Chlordecone intoxication in man. Neurology 28:626, 1978.

28. VESSEL, E. - Why are toxic reactions to drugs so often undetected initially? N. Engl. I. Med. 308:1027, 1980.

29. WERNECK, L.C. \& NOVAK, E.M. - Intoxicaçōes por inseticidads: microdissecçāo de nervos e histoquimica de músculos em 10 casos. Arq. Neuro-Psiquiat. (São Paulo) $37: 15,1979$.

30. W.H.O. Publication - Environmental Health-Criteria: 18. Arsenic. International Programe on Chemical Safety. W.H.O., Geneva, 1981.

31. W.H.O. Publication - Environmental Health-Criteria 29: 2,4 dichlorophenoxyacetic acid (2,4-D). International Programme on Chemical Safety. W.H.O., Geneva, 1984.

32. ZADMKOFF, C.M. - Toxic encephalopathy associated with use of insect repllant. J. Pediatrics $95: 140,1979$.

Departamento de Neurologia e Psiquiatria - Faculdade de Medicina de Botucatu, UNESP - 18600 - Botucatu, SP - Brasil. 